\title{
The Dynamics of a Delayed Predator-Prey Model with Double Allee Effect
}

\author{
Boli Xie, ${ }^{1,2}$ Zhijun Wang, ${ }^{1}$ Yakui Xue, ${ }^{2}$ and Zhenmin Zhang ${ }^{2}$ \\ ${ }^{1}$ School of Mechatronic Engineering, North University of China, Taiyuan, Shanxi 030051, China \\ ${ }^{2}$ Department of Mathematics, North University of China, Taiyuan, Shanxi 030051, China
}

Correspondence should be addressed to Boli Xie; boli.xie@163.com

Received 30 June 2015; Accepted 27 July 2015

Academic Editor: Carlo Bianca

Copyright (c) 2015 Boli Xie et al. This is an open access article distributed under the Creative Commons Attribution License, which permits unrestricted use, distribution, and reproduction in any medium, provided the original work is properly cited.

\begin{abstract}
We study the dynamics of a delayed predator-prey model with double Allee effect. For the temporal model, we showed that there exists a threshold of time delay in predator-prey interactions; when time delay is below the threshold value, the positive equilibrium $E^{*}$ is stable. However, when time delay is above the threshold value, the positive equilibrium $E^{*}$ is unstable and period solution will emerge. For the spatiotemporal model, through numerical simulations, we show that the model dynamics exhibit rich parameter space Turing structures. The obtained results show that this system has rich dynamics; these patterns show that it is useful for a delayed predator-prey model with double Allee effect to reveal the spatial dynamics in the real model.
\end{abstract}

\section{Introduction}

The Allee effect, named after the ecologist Warder Clyde Allee, is a phenomenon in biology characterized by a correlation between population size or density and the mean individual fitness of a population or species [1]. Allee effect can occur whenever fitness of an individual in a small or sparse population decreases as the population size or density also declines $[2,3]$. Allee effect contains two main types: strong Allee effect and weak Allee effect. A population exhibiting a weak Allee effect will possess a reduced per capita growth rate (directly related to individual fitness of the population) at lower population density or size. However, even at this low population size or density, the population will always exhibit a positive per capita growth rate. Meanwhile, a population exhibiting a strong Allee effect will have a critical population size or density under which the population growth rate becomes negative. Therefore, when the population density or size hits a number below this threshold, the population will be doomed.

There have been a large group of papers on predatorprey systems with Allee effect [4-12]. The most usual simple mathematical example of an Allee effect is given by the equation

$$
\frac{d N}{d T}=r N\left(1-\frac{N}{K}\right)(N-m),
$$

where $N$ denotes the population density, $r$ is the intrinsic rate of increase, $K$ is the carrying capacity, and $m$ is threshold of the Allee effect. The population has a negative growth rate for $N<m$ and a positive growth rate for $N>m$. If $m>0$, (1) is a strong Allee effect; if $m \leq 0$, (1) is a weak Allee effect. However, two mechanisms of Allee effects acting in the same population interact to produce an overall demographic Allee effect in a prey-predator interaction model which can also be common and can be complex [13] and their combined influence is termed as double.

There are also some works done on predator-prey systems with double Allee effect [14-16]. González-Olivares et al. found that the Gause-type predator-prey model with Allee effect can induce two limit cycles when the Allee effect is either strong or weak [14]. Huincahue-Arcos and GonzálezOlivares found that the Rosenzweig-MacArthur predation 
model with double Allee effects may be expressed by different mathematical formalizations; with the form used here, the existence of one limit cycle surrounding a positive equilibrium point is proved [15]. Pal and Saha found that the ratio dependent prey-predator system with a double Allee effect exhibits the bistability and there exists separatrix curve(s) in the phase plane implying that dynamics of the system are very sensitive to the variation of the initial conditions [16]. However, these previous works did not take into account the effect of space.

Time delay plays an important role in many biological dynamical systems, where time delays have been recognized to contribute critically to the outcome for prey densities under predation being stable or unstable [17]. Time delay due to gestation is included in some predator-prey models, because generally a duration of $\tau$ time units elapses between the time when an individual prey is killed and the moment when a corresponding increase in the predator population is realized [18]. Furthermore, time delays can be used to introduce oscillations $[19,20]$.

In the present study, our objective is to investigate a predator-prey model with double Allee effect and time delay. More specifically, the primary objective of the present study is to investigate the spatial patterns.

\section{Analysis of Temporal Model}

In this section, we consider a predator-prey model where the prey population growth is affected by double Allee effects with time delay. The following predator-prey model with double Allee effect has been proposed and studied [16]:

$$
\begin{aligned}
& \frac{d x}{d \widetilde{t}}=\frac{r x}{x+n}\left(1-\frac{x}{K}\right)(x-m)-\frac{c x y}{x+\vartheta y}, \\
& \frac{d y}{d \widetilde{t}}=\frac{c_{1} x y}{x+\vartheta y}-d y,
\end{aligned}
$$

where $x$ and $y$ stand for prey and predator density, $r$ is the intrinsic rate of increase, $K$ is the carrying capacity, $m$ is threshold of the Allee effect, $c$ stands for capturing rate of the predator, $\vartheta$ stands for half capturing saturation constant, $c_{1}$ stands for conversion rate of prey into predators biomass, and $d$ stands for natural death rate of predator.

Following [16], through a nondimensional transformation,

$$
\begin{aligned}
& u=\frac{1}{K} x, \\
& v=\frac{\vartheta}{K} y, \\
& t=r \tilde{t},
\end{aligned}
$$

we arrive at the following equations:

$$
\begin{aligned}
& \frac{d u}{d t}=\frac{u(1-u)(u-\gamma)}{u+\theta}-\frac{\alpha u v}{u+v}, \\
& \frac{d v}{d t}=\frac{\beta u v}{u+v}-\delta v,
\end{aligned}
$$

where

$$
\begin{aligned}
& \theta=\frac{n}{K}, \\
& \gamma=\frac{m}{K}, \\
& \alpha=\frac{c}{r \vartheta}, \\
& \beta=\frac{c_{1}}{r}, \\
& \delta=\frac{d}{r} .
\end{aligned}
$$

In the section, our objective is to investigate the predatorprey model with double Allee effect and time delay. The model is given by

$$
\begin{aligned}
& \frac{d u}{\partial t}=\frac{u(1-u)(u-\gamma)}{u+\theta}-\frac{\alpha u v(t-\tau)}{u+v(t-\tau)}, \\
& \frac{d v}{\partial t}=\frac{\beta u(t-\tau) v}{u(t-\tau)+v}-\delta v,
\end{aligned}
$$

where $\tau>0$ is a constant delay due to gestation.

We analyze model (6) under the initial conditions

$$
\begin{aligned}
& u(0)>0, \\
& v(0)>0 .
\end{aligned}
$$

Next, we will discuss the dynamics of model (6). We determined that model (6) and model (4) have two boundary equilibria named $E_{0}=(1,0)$ and $E_{1}=(\gamma, 0)$ and a unique positive equilibrium named $E^{*}=\left(u^{*}, v^{*}\right)$, where

$$
\begin{aligned}
& u^{*}=\frac{\beta-\alpha \beta+\delta \alpha+\gamma \beta+\sqrt{(\beta-\alpha \beta+\delta \alpha)^{2}+4 \beta \theta \alpha \delta}}{2 \beta}, \\
& v^{*}=\frac{(\beta-\delta) u^{*}}{\delta} .
\end{aligned}
$$

We aim to look for the conditions so that $\left(u^{*}, v^{*}\right)$ is stable for the temporal model and is unstable for the spatiotemporal model. We always assume that $\left(u^{*}, v^{*}\right)$ is linearly stable with respect to the perturbation of $u$ and $v$; thus, the eigenvalues of the Jacobian 


$$
J=\left(\begin{array}{ll}
a_{11} & a_{12} \\
a_{21} & a_{22}
\end{array}\right)=\left(\begin{array}{cc}
\frac{-3\left(u^{*}\right)^{2}+2 u^{*} \gamma+2 u^{*}-\gamma}{u^{*}+\theta}-\frac{u^{*}\left(1-u^{*}\right)\left(u^{*}-\gamma\right)}{\left(u^{*}+\theta\right)^{2}}-\frac{\alpha(\beta-\delta)^{2}}{\beta^{2}} & -\frac{\alpha \delta^{2}}{\beta^{2}} \\
\frac{(\beta-\delta)^{2}}{\beta} & -\frac{(\beta-\delta) \delta}{\beta}
\end{array}\right)
$$

at $\left(u^{*}, v^{*}\right)$ must have negative real parts, which is equivalent to

$$
\begin{aligned}
\operatorname{tr}(J) & =a_{11}+a_{22}<0, \\
\operatorname{det}(J) & =a_{11} a_{22}-a_{12} a_{21}>0 .
\end{aligned}
$$

Next, we consider small spatiotemporal perturbations $h$ and $p$ on a homogeneous steady state $E^{*}\left(u^{*}, v^{*}\right)$. Let $h=u-$ $u^{*}$ and $p=v-v^{*}$; then, we derive that

$$
\begin{aligned}
& \frac{d h}{\partial t}=a_{11} h+a_{12} p(t-\tau), \\
& \frac{d p}{\partial t}=a_{21} h(t-\tau)+a_{22} p .
\end{aligned}
$$

Spatiotemporal perturbations $h$ and $p$ are given by

$$
\begin{aligned}
& h=h^{*} e^{\lambda t} \cos \left(k_{x} x\right), \\
& p=p^{*} e^{\lambda t} \cos \left(k_{x} x\right) .
\end{aligned}
$$

By substitution of this form in (11), we get the following matrix equation about eigenvalues:

$$
\left(\begin{array}{cc}
\lambda-a_{11} & -a_{12} e^{-\lambda \tau} \\
-a_{21} e^{-\lambda \tau} & \lambda-a_{22}
\end{array}\right)\left(\begin{array}{l}
h^{*} \\
p^{*}
\end{array}\right)=\left(\begin{array}{l}
0 \\
0
\end{array}\right) .
$$

Linear system (11) is characterized by the equation

$$
\lambda^{2}-\left(a_{11}+a_{22}\right) \lambda+a_{11} a_{22}-a_{12} a_{21} e^{-2 \lambda \tau}=0 .
$$

If $\lambda=i \omega$ is a root of (14), then we have

$$
\begin{aligned}
-\omega^{2}+a_{11} a_{22} & =a_{12} a_{21} \cos (2 \omega \tau), \\
\left(a_{11}+a_{22}\right) \omega & =a_{12} a_{21} \sin (2 \omega \tau),
\end{aligned}
$$

which leads to

$$
\omega^{4}+\left(a_{11}^{2}+a_{22}^{2}\right) \omega^{2}+a_{11}^{2} a_{22}^{2}-a_{12}^{2} a_{21}^{2}=0 .
$$

Then, (16) has the solution

$$
\begin{aligned}
& \omega_{c}^{2} \\
& =\frac{-\left(a_{11}^{2}+a_{22}^{2}\right)+\sqrt{\left(a_{11}^{2}+a_{22}^{2}\right)^{2}-4\left(a_{11}^{2} a_{22}^{2}-a_{12}^{2} a_{21}^{2}\right)}}{2} .
\end{aligned}
$$

From (15), we can obtain

$$
\tau_{c}=\frac{1}{2 \omega_{c}} \arccos \frac{-\omega_{c}^{2}+a_{11} a_{22}}{a_{12} a_{21}} .
$$

Now, we investigate the sign of $\left.(d \operatorname{Re}(\lambda) / d \tau)\right|_{\tau=\tau_{c}}$. Let $\lambda=$ $\sigma+i \omega$ be a solution of (14); then

$$
\begin{aligned}
\operatorname{Re}(\lambda)= & \sigma^{2}-\omega^{2}-\left(a_{11}+a_{22}\right) \sigma+a_{11} a_{22} \\
& -a_{12} a_{21} e^{-2 \sigma \tau} \cos (2 \omega \tau)=0, \\
\operatorname{Im}(\lambda)= & 2 \sigma \omega-\left(a_{11}+a_{22}\right) \omega+a_{12} a_{21} e^{-2 \sigma \tau} \sin (2 \omega \tau) \\
= & 0 .
\end{aligned}
$$

By derivation of $\tau$ in both sides of (19), notice that $\lambda=\sigma+i \omega=$ $i \omega$; we can get

$$
\begin{aligned}
& A \frac{d \sigma}{d \tau}-B \frac{d \omega}{d \tau}+C=0, \\
& A \frac{d \sigma}{d \tau}+B \frac{d \omega}{d \tau}+D=0,
\end{aligned}
$$

where

$$
\begin{aligned}
& A=-\left(a_{11}+a_{22}\right)+2 \tau a_{12} a_{21} \cos (2 \omega \tau), \\
& B=2 \omega-2 \tau a_{12} a_{21} \sin (2 \omega \tau), \\
& C=2 \omega a_{12} a_{21} \sin (2 \omega \tau), \\
& D=2 \omega a_{12} a_{21} \cos (2 \omega \tau) .
\end{aligned}
$$

Thus, we can get

$$
\left.\frac{d \operatorname{Re}(\lambda)}{d \tau}\right|_{\tau=\tau_{c}}=\left.\frac{d \sigma}{d \tau}\right|_{\tau=\tau_{c}}=-\frac{A C+B D}{A^{2}+B^{2}} .
$$

Binding (15) and (16), we obtain

$$
-(A C+B D)=2 \omega^{2}\left(a_{11}^{2}+a_{22}^{2}+2 \omega^{2}\right)>0,
$$

which implies that $\left.(d \operatorname{Re}(\lambda) / d \tau)\right|_{\tau=\tau_{c}}>0$. Furthermore, we get the following conclusions: If the delay $\tau$ is satisfied $\tau \neq 0$, then system (6) exhibits a Hopf bifurcation critical $\tau=\tau_{c}$. When $\tau<\tau_{c}$, the positive equilibrium $E^{*}$ is stable, but when $\tau>\tau_{c}$, the positive equilibrium $E^{*}$ is unstable and period solution will emerge.

We take the following values: $\alpha=0.6, \beta=0.8, \gamma=-0.3$, $\delta=0.58$, and $\theta=2.2$. Through calculations, we obtain the critical value $\tau_{c}=0.7397$; then $E^{*}=(0.36,0.1365517241)$. The initial value is $(0.3,0.1)$.

We adopt $\tau=0.2<\tau_{c}$. From Figure 1 , we can see that the positive equilibrium $E^{*}$ is stable.

We adopt $\tau=0.81>\tau_{c}$. From Figure 2, we can see that the positive equilibrium $E^{*}$ is unstable. 

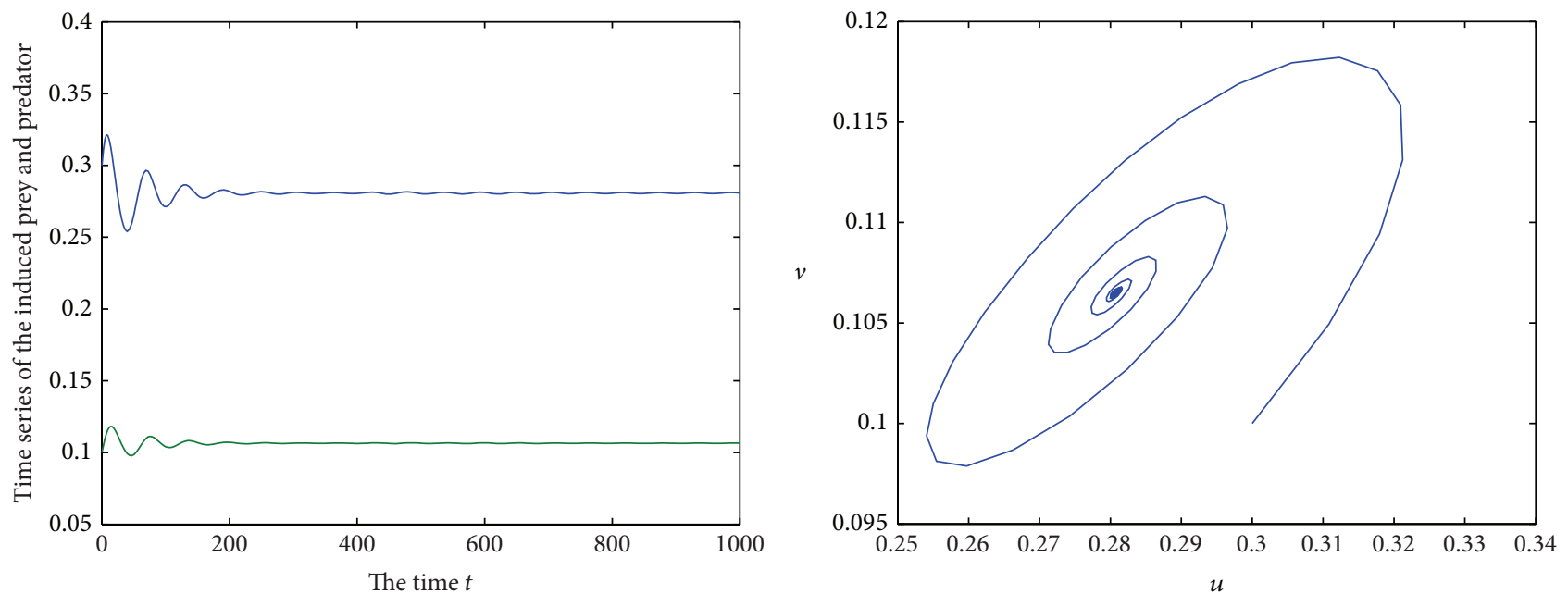

$-v$

(a)

(b)

Figure 1: Behavior and phase portrait of system (6). Parameter values are used as $\alpha=0.6, \beta=0.8, \gamma=-0.3, \delta=0.58, \theta=2.2$, and $\tau=0.2<\tau_{c}$.

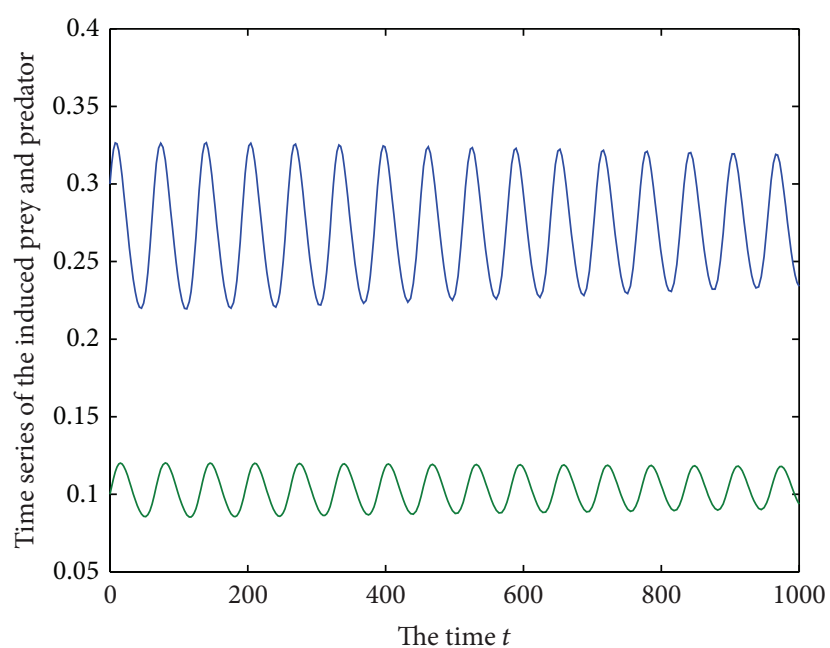

$-u$

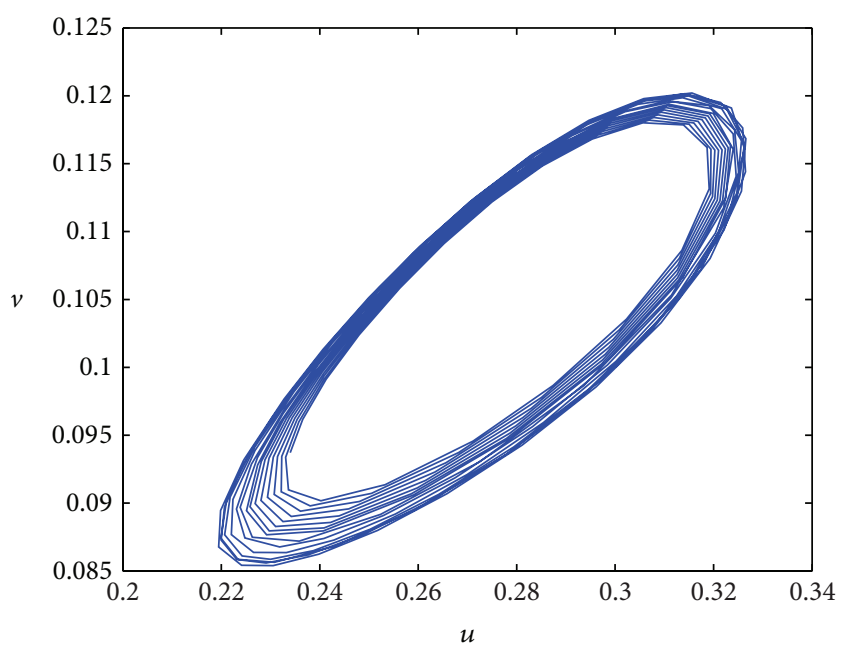

(b)

Figure 2: Behavior and phase portrait of system (6). Parameter values are used as $\alpha=0.6, \beta=0.8, \gamma=-0.3, \delta=0.58, \theta=2.2$, and $\tau=0.81>\tau_{c}$.

\section{Analysis of Spatiotemporal Model}

In the section, our objective is to consider the spatiotemporal system with double Allee effect and time delay:

$$
\begin{aligned}
& \frac{\partial u}{\partial t}=\frac{u(1-u)(u-\gamma)}{u+\theta}-\frac{\alpha u v(t-\tau)}{u+v(t-\tau)}+d_{1} \nabla^{2} u, \\
& \frac{\partial v}{\partial t}=\frac{\beta u(t-\tau) v}{u(t-\tau)+v}-\delta v+d_{2} \nabla^{2} v .
\end{aligned}
$$

Similar to the analysis of (6), we consider small spatiotemporal perturbations $h=u-u^{*}$ and $p=v-v^{*}$ on a homogeneous steady state $E^{*}\left(u^{*}, v^{*}\right)$. The linearized system takes the form

$$
\begin{aligned}
& \frac{\partial h}{\partial t}=a_{11} h+a_{12} p(t-\tau)+d_{1} \nabla^{2} h, \\
& \frac{\partial p}{\partial t}=a_{21} h(t-\tau)+a_{22} p+d_{2} \nabla^{2} p .
\end{aligned}
$$




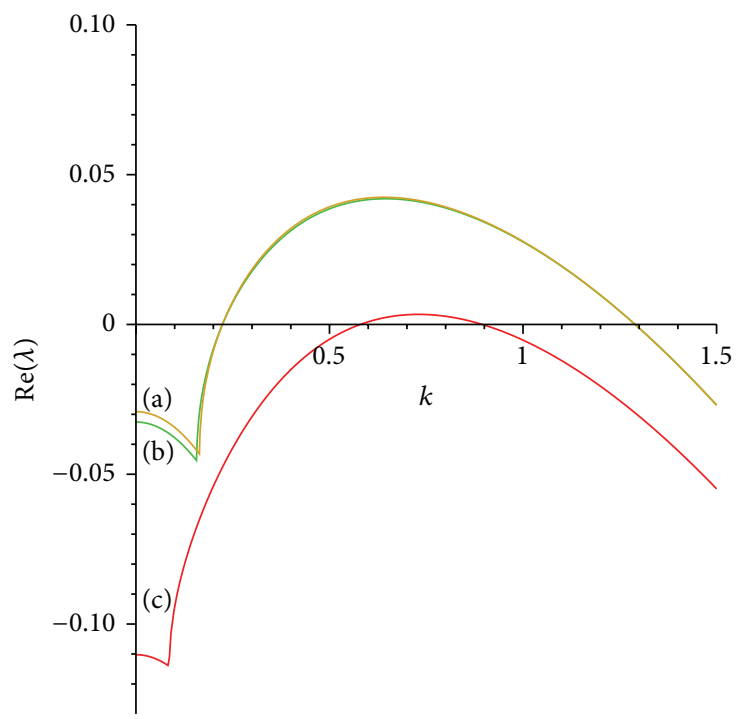

Figure 3: An illustration of model (24). We set the parameter values as (a) $\alpha=0.6, \beta=0.8, \gamma=-0.3, \delta=0.58, \theta=2.2, d_{1}=0.08, d_{2}=1$, and $\tau=0.2$; (b) $\alpha=0.6, \beta=0.8, \gamma=-0.3, \delta=0.58, \theta=2.2, d_{1}=0.08, d_{2}=1$, and $\tau=0.02$; (c) $\alpha=0.6, \beta=1.5, \gamma=-0.3, \delta=1.1, \theta=2.2$, $d_{1}=0.05, d_{2}=1$, and $\tau=0.02$.

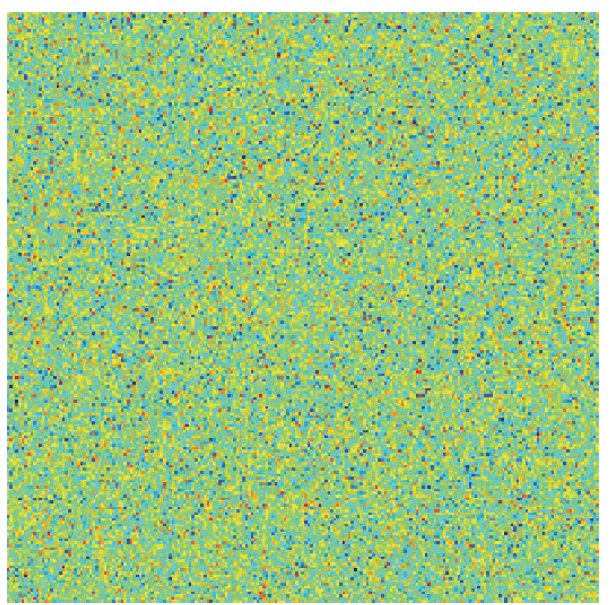

(a)

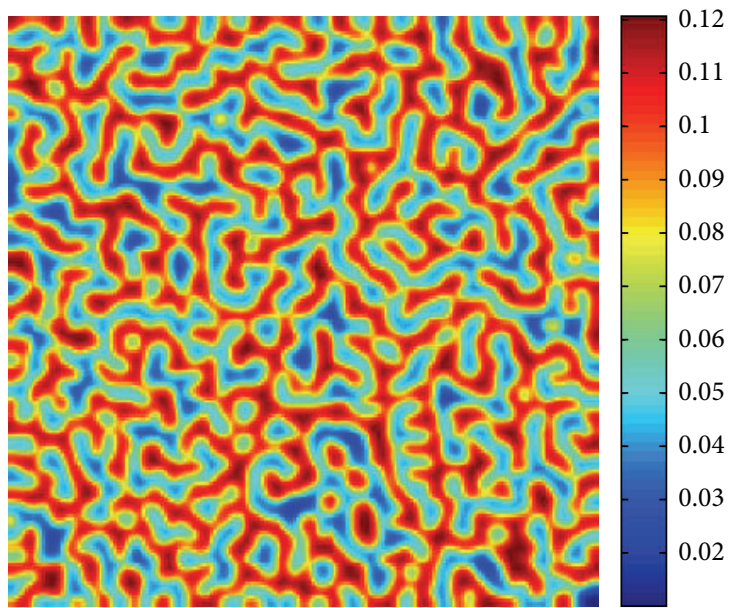

(c)
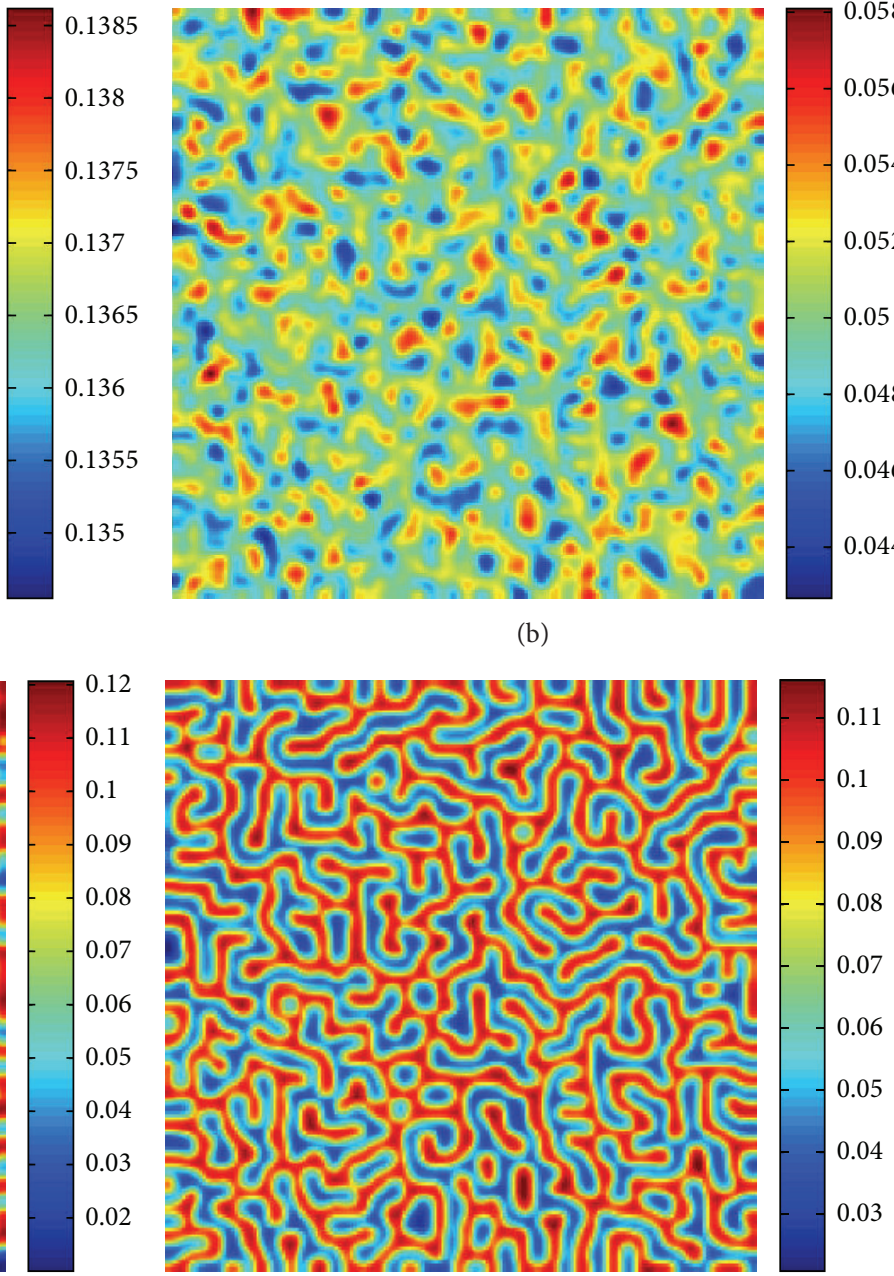

(d)

Figure 4: Snapshots of the time evolution of the prey at different instants with $\alpha=0.6, \beta=0.8, \gamma=-0.3, \delta=0.58, \theta=2.2, d_{1}=0.08, d_{2}=1$, and $\tau=0.2$, which are in the Turing space. (a) 0 iterations; (b) 5000 iterations; (c) 10000 iterations; (d) 100000 iterations. 


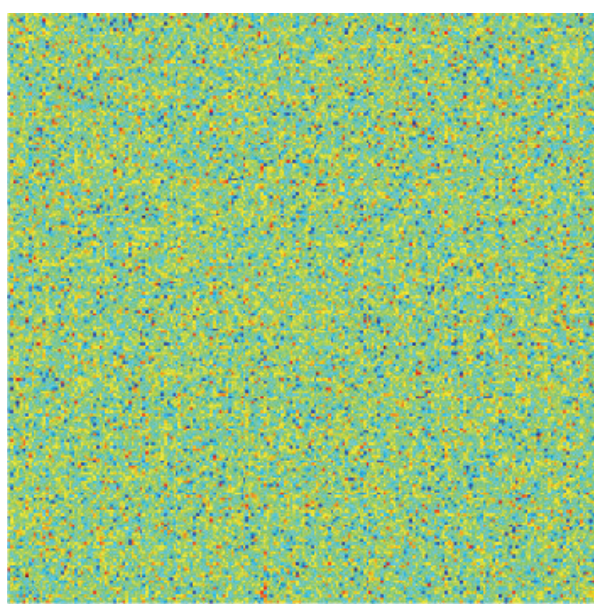

(a)

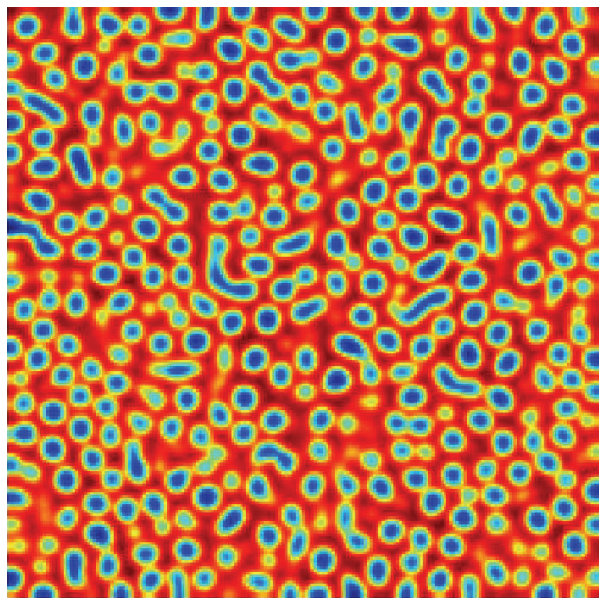

(c)
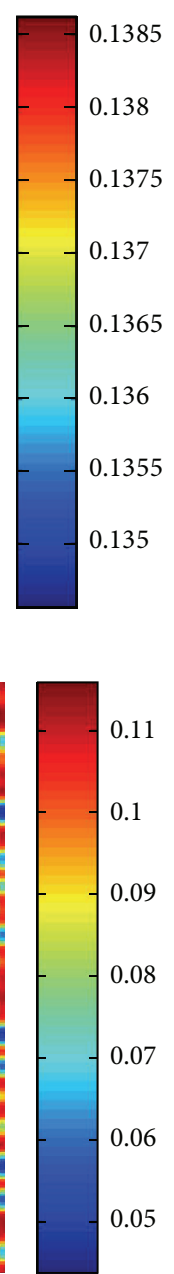

0.05
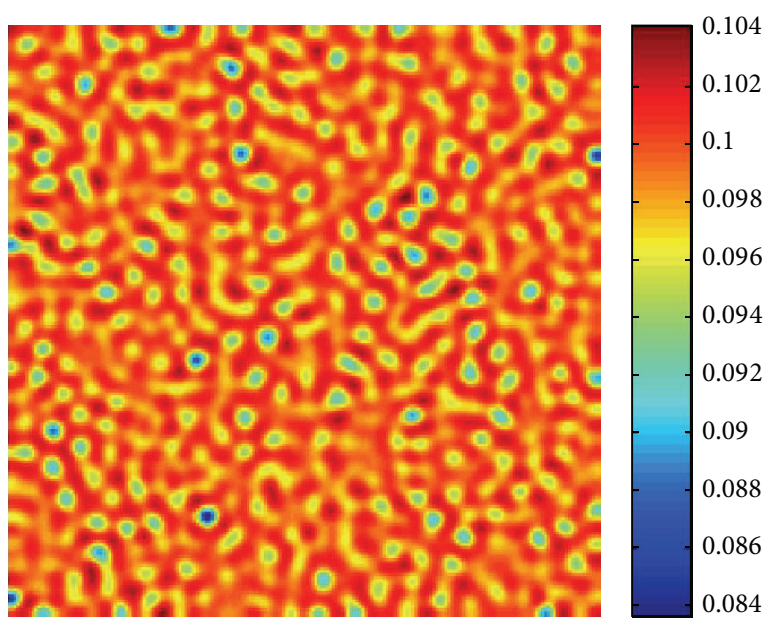

(b)

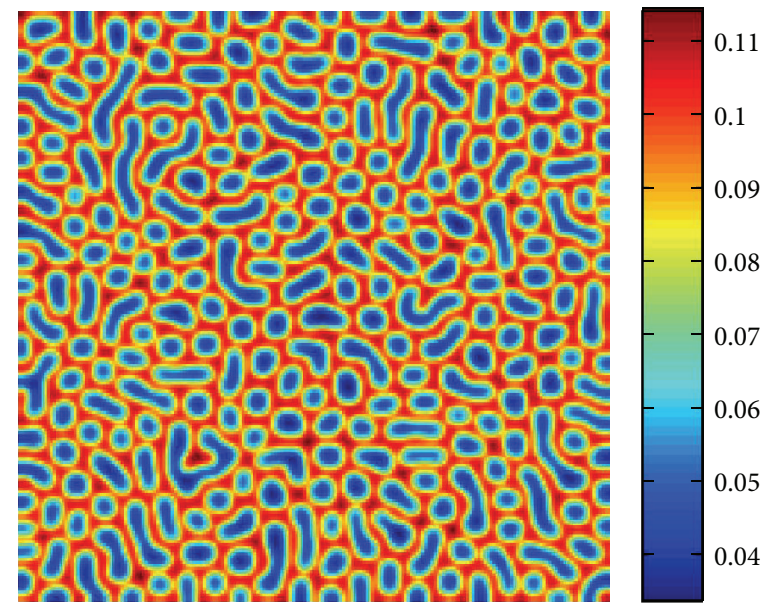

(d)

FIGURE 5: Snapshots of the time evolution of the prey at different instants with $\alpha=0.6, \beta=0.8, \gamma=-0.3, \delta=0.58, \theta=2.2, d_{1}=0.08, d_{2}=1$, and $\tau=0.02$, which are in the Turing space. (a) 0 iterations; (b) 10000 iterations; (c) 15000 iterations; (d) 100000 iterations.

By substitution of form (12) in (25), we get the following matrix equation about eigenvalues:

$$
\left(\begin{array}{cc}
\lambda-a_{11}+d_{1} k^{2} & -a_{12} e^{-\lambda \tau} \\
-a_{21} e^{-\lambda \tau} & \lambda-a_{22}+d_{2} k^{2}
\end{array}\right)\left(\begin{array}{l}
h^{*} \\
p^{*}
\end{array}\right)=\left(\begin{array}{l}
0 \\
0
\end{array}\right) .
$$

The characteristic equation for the linear system (25) is given by

$$
\begin{aligned}
\lambda^{2}- & {\left[\left(a_{11}-d_{1} k^{2}\right)+\left(a_{22}-d_{2} k^{2}\right)\right] \lambda } \\
& +\left(a_{11}-d_{1} k^{2}\right)\left(a_{22}-d_{2} k^{2}\right)-a_{12} a_{21} e^{-2 \lambda \tau}=0 .
\end{aligned}
$$

Spatial patterns form if (27) has root $\lambda=i \omega$, which are called delay-driven spatial patterns. Moreover, the critical value of the delay $\tau$ is called the Turing bifurcation. If $i \omega$ is a root of (27), then we have

$$
\begin{aligned}
-\omega^{2}+\left(a_{11}-d_{1} k^{2}\right)\left(a_{22}-d_{2} k^{2}\right) & =a_{12} a_{21} \cos (2 \omega \tau), \\
{\left[\left(a_{11}-d_{1} k^{2}\right)+\left(a_{22}-d_{2} k^{2}\right)\right] \omega } & =a_{12} a_{21} \sin (2 \omega \tau),
\end{aligned}
$$

which leads to

$$
\omega^{4}+\alpha_{k} \omega^{2}+\beta_{k}=0
$$

where

$$
\begin{aligned}
& \alpha_{k}=\left(a_{11}-d_{1} k^{2}\right)^{2}+\left(a_{22}-d_{2} k^{2}\right)^{2} \\
& \beta_{k}=\left(a_{11}-d_{1} k^{2}\right)^{2}\left(a_{22}-d_{2} k^{2}\right)^{2}-a_{12}^{2} a_{21}^{2} .
\end{aligned}
$$

Then, (29) has the solution

$$
\omega_{\mathrm{sc}}^{2}=\frac{-\alpha_{k}+\sqrt{\alpha_{k}^{2}-4 \beta_{k}}}{2} .
$$

From (28), we can obtain

$$
\tau_{\mathrm{sc}}=\frac{1}{2 \omega_{\mathrm{sc}}} \arccos \frac{-\omega_{\mathrm{sc}}^{2}+\left(a_{11}-d_{1} k^{2}\right)\left(a_{22}-d_{2} k^{2}\right)}{a_{12} a_{21}} .
$$




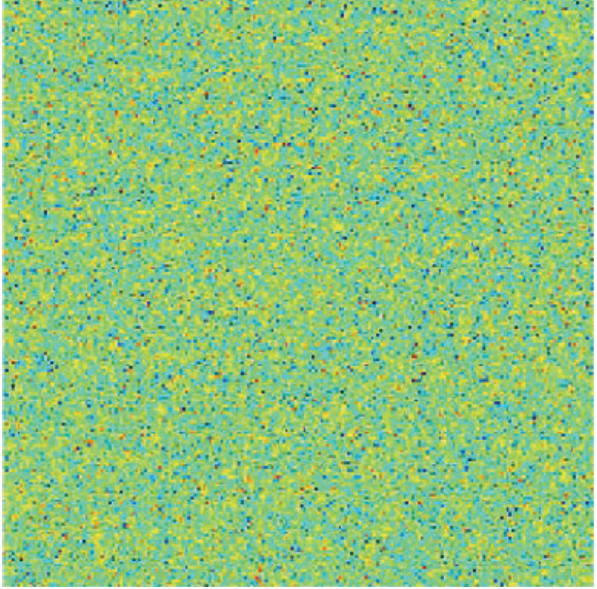

(a)

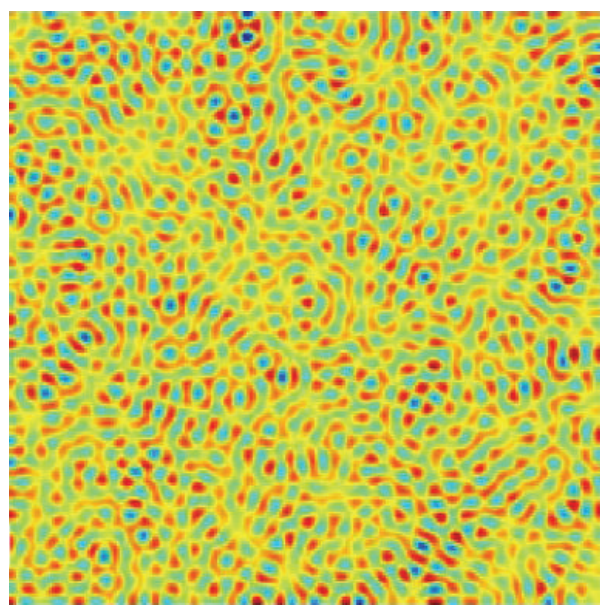

(c)
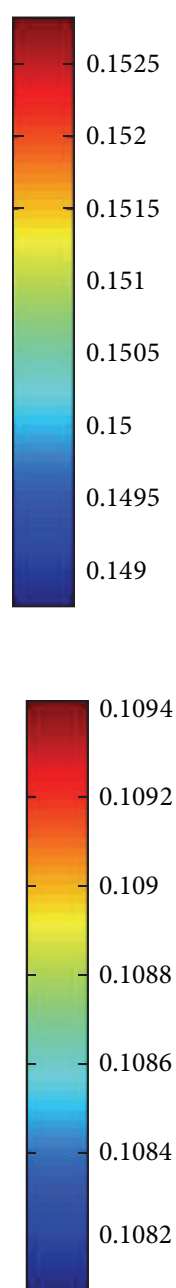

0.1082

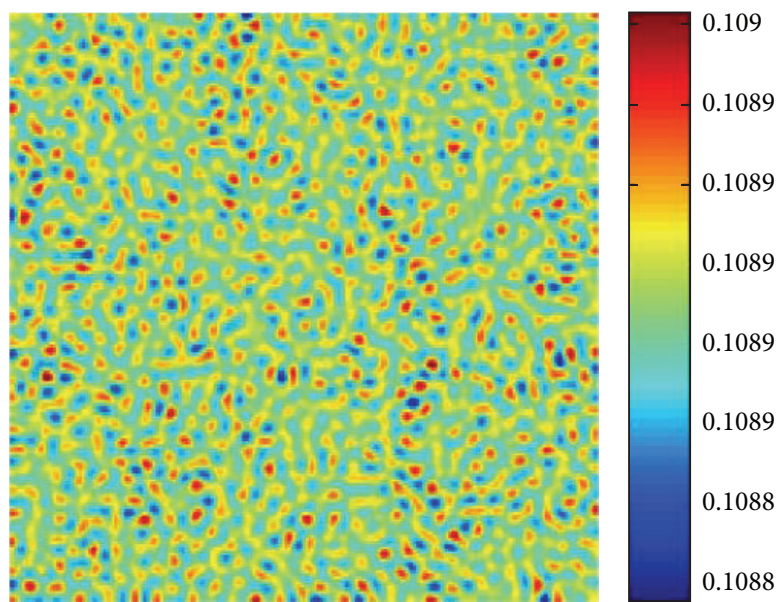

(b)

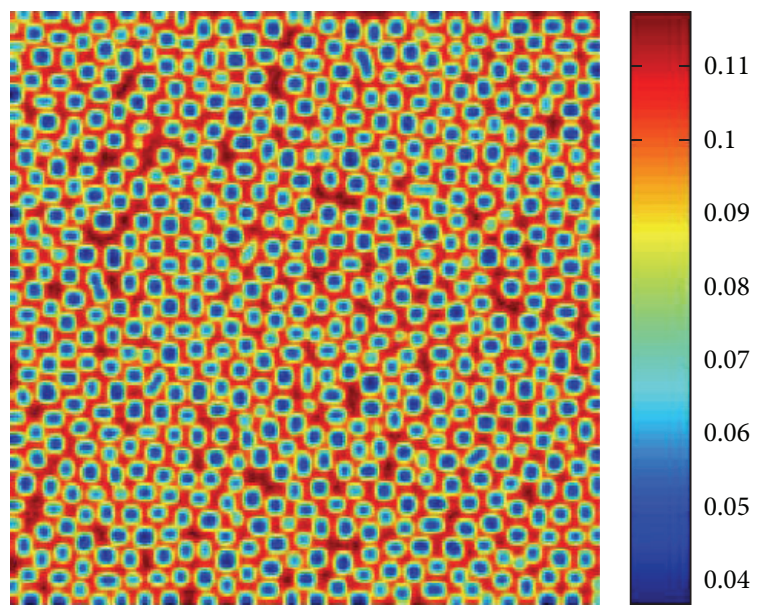

(d)

FIGURE 6: Snapshots of the time evolution of the prey at different instants with $\alpha=0.6, \beta=1.5, \gamma=-0.3, \delta=1.1, \theta=2.2, d_{1}=0.08, d_{2}=1$, and $\tau=0.02$, which are in the Turing space. (a) 0 iterations; (b) 10000 iterations; (c) 50000 iterations; (d) 100000 iterations.

Using mathematical calculations, a Turing bifurcation is produced when the following conditions are met:

$$
\begin{aligned}
& \operatorname{Im}(\lambda)=0, \\
& \operatorname{Re}(\lambda)=0, \\
& \theta_{T}=\frac{1}{4} \frac{\beta^{2}-2 \alpha \beta^{2}+2 \beta \delta \alpha-2 \gamma \beta^{2}+\alpha^{2} \beta^{2}-2 \alpha^{2} \beta \delta-2 \alpha \beta^{2} \gamma+\delta^{2} \alpha^{2}+2 \delta \alpha \gamma \beta+\gamma^{2} \beta^{2}}{\alpha \beta(\beta-\delta)} .
\end{aligned}
$$

To see well the effect of cross diffusion and time delay, we plot the dispersion relation keeping the parameter values fixed in Figure 3. It can be seen from Figure 3 that Turing modes $\operatorname{Re}(\lambda)>0$ can be available.

\section{Pattern Structures}

In the following, we will perform a series of numerical simulations on the two-dimensional model (24) using zero boundary conditions and $M \times N$ discrete lattice. 
For model (24), space and time were approximated using the finite difference method and Euler's method, taking the time step as $\Delta t=0.01$, the space step as $\Delta h=1$, and $M=$ $N=200$. The results indicated that $\Delta h$ and $\Delta t$ are reduced and do not lead to considerable changes in the results.

In Figure 4, we set $\alpha=0.6, \beta=0.8, \gamma=-0.3, \delta=0.58$, $\theta=2.2, d_{1}=0.08, d_{2}=1$, and $\tau=0.2$. In this case, the infected populations exhibit stationary labyrinthine patterns.

In Figure 5, we set $\alpha=0.6, \beta=0.8, \gamma=-0.3, \delta=0.58$, $\theta=2.2, d_{1}=0.08, d_{2}=1$, and $\tau=0.02$. We can see that short stripe-like pattern and spotted patterns emerge coexist.

In Figure 6, we set $\alpha=0.6, \beta=1.5, \gamma=-0.3, \delta=1.1$, $\theta=2.2, d_{1}=0.05, d_{2}=1$, and $\tau=0.02$. As time passes, regular spotted patterns appear in space, and the dynamics of the system do not undergo any further changes.

\section{Discussions}

In this paper, the dynamics of a delayed predator-prey model with double Allee effect were considered. First, we discuss the temporal model (6); we showed that there exists a Hopf bifurcation threshold $\tau_{c}$ of time delay; when $\tau<\tau_{c}$, the positive equilibrium $E^{*}$ of system (6) is stable. However, when $\tau>\tau_{c}$, the positive equilibrium $E^{*}$ of system (6) is unstable and period solution will emerge. Second, we discuss the spatiotemporal model (24); the spatial patterns via numerical simulations are illustrated, which show that the model dynamics exhibit rich parameter space Turing structures.

Although more work is needed, in principle, it seems that delay and diffusion are able to generate many different kinds of spatiotemporal patterns. For such reasons, we can predict that delay and diffusion can be considered as an important mechanism for the appearance of complex spatiotemporal dynamics in other models, such as predator-prey model and mutualistic model.

\section{Conflict of Interests}

The authors declare that there is no conflict of interests regarding the publication of this paper.

\section{Acknowledgments}

This work is supported by the National Sciences Foundation of China (10471040) and the National Sciences Foundation of Shanxi Province (2009011005-1).

\section{References}

[1] F. Courchamp, J. Berec, and J. Gascoigne, Allee Effects in Ecology and Conservation, Oxford University Press, Oxford, UK, 2008.

[2] F. Courchamp, T. Clutton-Brock, and B. Grenfell, "Inverse density dependence and the Allee effect," Trends in Ecology \& Evolution, vol. 14, no. 10, pp. 405-410, 1999.

[3] P. A. Stephens and W. J. Sutherland, "Consequences of the Allee effect for behaviour, ecology and conservation," Trends in Ecology and Evolution, vol. 14, no. 10, pp. 401-405, 1999.
[4] S. V. Petrovskii, A. Y. Morozov, and E. Venturino, "Allee effect makes possible patchy invasion in a predator-prey system," Ecology Letters, vol. 5, no. 3, pp. 345-352, 2002.

[5] A. Morozov, S. Petrovskii, and B.-L. Li, "Bifurcations and chaos in a predator-prey system with the Allee effect," Proceedings of the Royal Society B: Biological Sciences, vol. 271, no. 1546, pp. 1407-1414, 2004.

[6] A. Morozov, S. Petrovskii, and B.-L. Li, "Spatiotemporal complexity of patchy invasion in a predator-prey system with the Allee effect," Journal of Theoretical Biology, vol. 238, no. 1, pp. 18-35, 2006.

[7] D. Hadjiavgousti and S. Ichtiaroglou, "Allee effect in a preypredator system," Chaos, Solitons \& Fractals, vol. 36, no. 2, pp. 334-342, 2008.

[8] C. Celik and O. Duman, "Allee effect in a discrete-time predatorprey system," Chaos, Solitons and Fractals, vol. 40, no. 4, pp. 1956-1962, 2009.

[9] A. Verdy, "Modulation of predator-prey interactions by the Allee effect," Ecological Modelling, vol. 221, no. 8, pp. 1098-1107, 2010.

[10] J. Wang, J. Shi, and J. Wei, "Predator-prey system with strong Allee effect in prey," Journal of Mathematical Biology, vol. 62, no. 3, pp. 291-331, 2011.

[11] L. Cai, G. Chen, and D. Xiao, "Multiparametric bifurcations of an epidemiological model with strong Allee effect," Journal of Mathematical Biology, vol. 67, no. 2, pp. 185-215, 2013.

[12] G.-Q. Sun, L. Li, Z. Jin, Z.-K. Zhang, and T. Zhou, "Pattern dynamics in a spatial predator-prey system with Allee effect," Abstract and Applied Analysis, vol. 2013, Article ID 921879, 12 pages, 2013.

[13] L. Berec, E. Angulo, and F. Courchamp, "Multiple Allee effects and population management," Trends in Ecology \& Evolution, vol. 22, no. 4, pp. 185-191, 2007.

[14] E. González-Olivares, B. González-Yaez, J. Mena Lorca, A. Rojas-Palma, and J. D. Flores, "Consequences of double Allee effect on the number of limit cycles in a predator-prey model," Computers \& Mathematics with Applications, vol. 62, no. 9, pp. 3449-3463, 2011.

[15] J. Huincahue-Arcos and E. González-Olivares, "The Rosenzweig-MacArthur predation model with double Allee effects on prey," in Proceedings of the International Conference on Applied Mathematics and Computational Methods in Engineering, pp. 206-211, 2013.

[16] P. J. Pal and T. Saha, "Qualitative analysis of a predator-prey system with double Allee effect in prey," Chaos, Solitons \& Fractals, vol. 73, pp. 36-63, 2015.

[17] A. F. Nindjin, M. A. Aziz-Alaoui, and M. Cadivel, "Analysis of a predator-prey model with modified Leslie-Gower and Hollingtype II schemes with time delay," Nonlinear Analysis: Real World Applications, vol. 7, no. 5, pp. 1104-1118, 2006.

[18] R. Xu and L. S. Chen, "Persistence and stability for a twospecies ratio-dependent predator-prey system with time delay in a two-patch environment," Computers \& Mathematics with Applications, vol. 40, no. 4-5, pp. 577-588, 2000.

[19] C. Bianca and L. Guerrini, "On the Dalgaard-Strulik model with logistic population growth rate and delayed-carrying capacity," Acta Applicandae Mathematicae, vol. 128, pp. 39-48, 2013.

[20] C. Bianca and L. Guerrini, "Existence of limit cycles in the Solow model with delayed-logistic population growth," The Scientific World Journal, vol. 2014, Article ID 207806, 8 pages, 2014. 


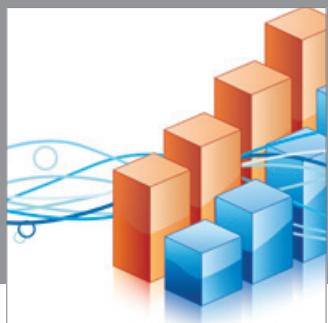

Advances in

Operations Research

mansans

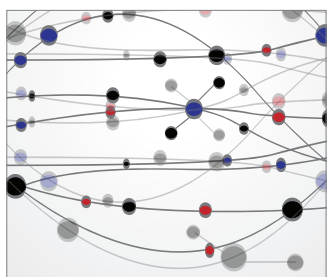

The Scientific World Journal
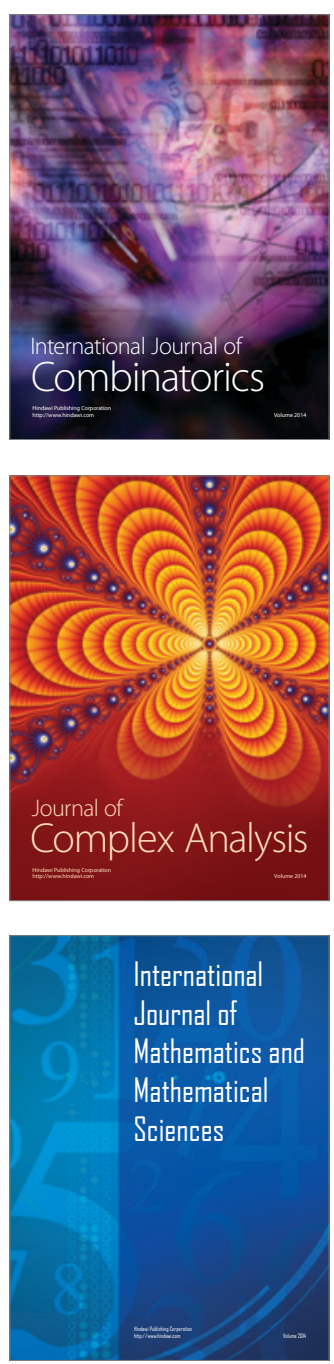
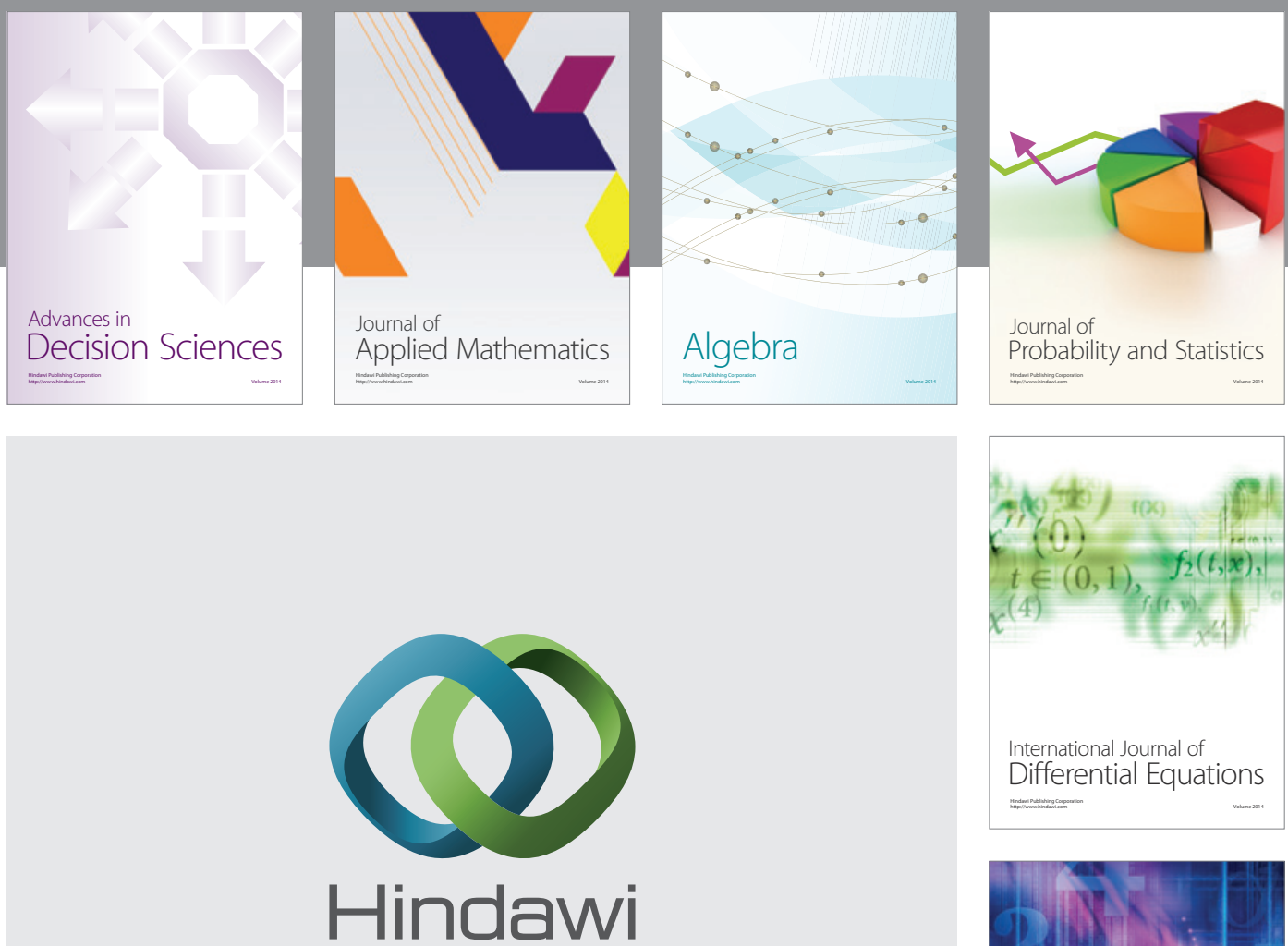

Submit your manuscripts at http://www.hindawi.com
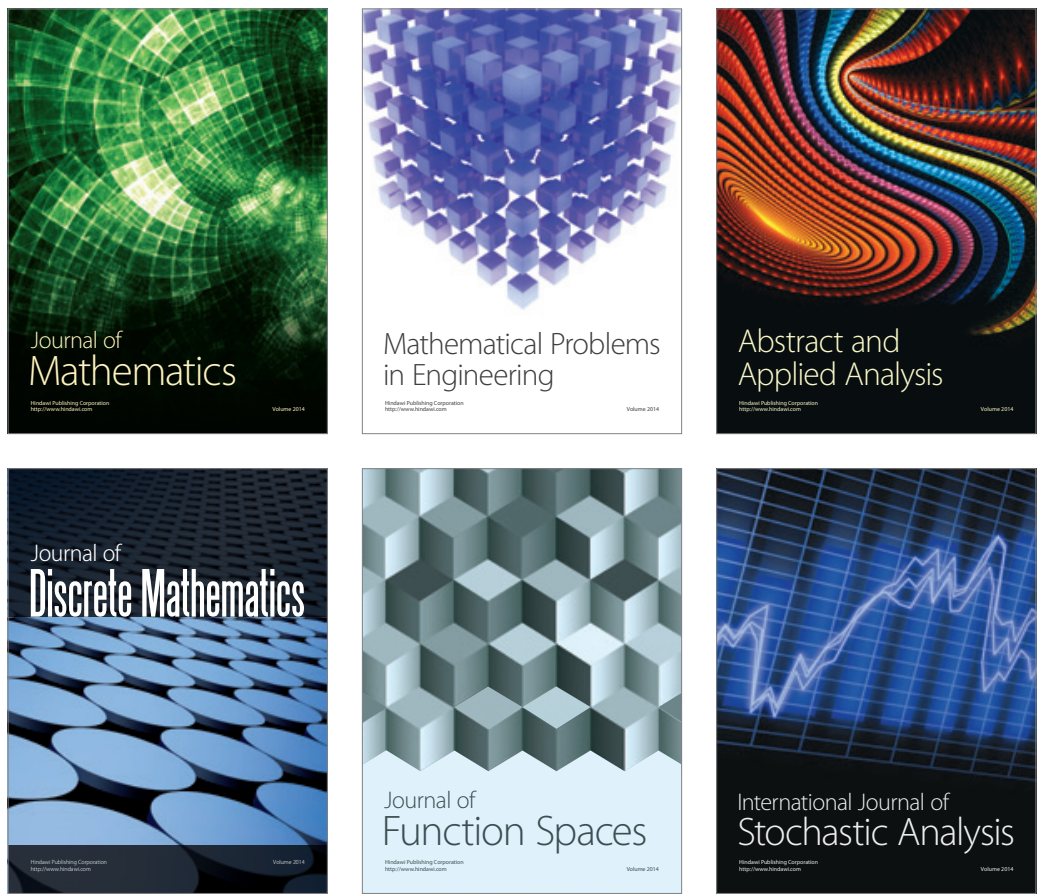

Journal of

Function Spaces

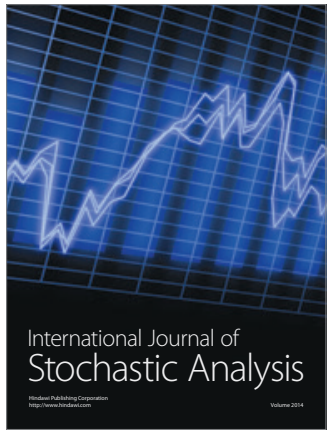

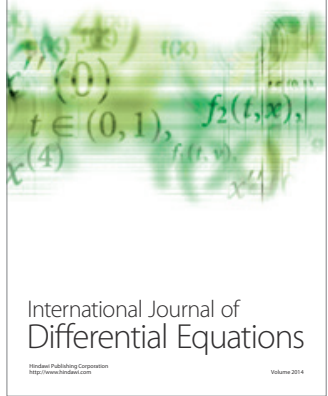
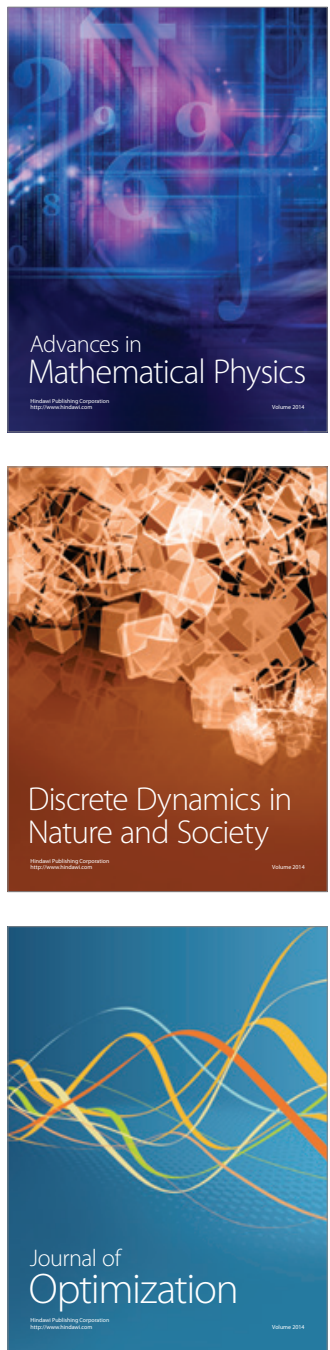